\title{
Time Synchronization and Performance of BeiDou Satellite Clocks in Orbit
}

\author{
Han Chunhao, Cai Zhiwu, Lin Yuting, Liu Li, Xiao Shenghong, \\ Zhu Lingfeng, and Wang Xianglei
}

Beijing Satellite Navigation Center, Beijing 100094, China

Correspondence should be addressed to Lin Yuting; lyt1108@163.com

Received 24 March 2013; Revised 3 July 2013; Accepted 31 July 2013

Academic Editor: Sandro Radicella

Copyright (C) 2013 Han Chunhao et al. This is an open access article distributed under the Creative Commons Attribution License, which permits unrestricted use, distribution, and reproduction in any medium, provided the original work is properly cited.

The time model of Beidou satellite clocks is analyzed. The general relations of satellite clocks with the system time are studied. The error sources of two-way radio time transfer between satellites and uplink stations are analyzed. The uncertainty of type A is about $0.3 \mathrm{~ns}$ in Beidou system. All the satellite clocks in orbit of Beidou satellite navigation system are evaluated by the clock offsets observed by the two-way radio time transfer. The frequency stabilities at a sample time of $10000 \mathrm{~s}$ and 1 day for all the satellite clocks are better than $1.0 \times 10^{-13}$. It means that the performance of Beidou satellite clocks in orbit is consistent with the ground test, and the results in orbit are a little better than those in ground vacuum.

\section{Introduction}

Beidou satellite navigation system began to provide regional service since December 2012. The constellation of Beidou system is constituted of 14 satellites in orbit: 5GEO satellites, 5IGSO satellites, and 4MEO satellites. Table 1 shows the basic information of the Beidou satellites. Service area now covers latitude $55^{\circ} \mathrm{S} \sim 55^{\circ} \mathrm{N}$ and longitude $55^{\circ} \mathrm{E} \sim 180^{\circ} \mathrm{E}$. Practical operational accuracy of Beidou system is better than $10 \mathrm{~m}$ (95\%) in horizontal and $15 \mathrm{~m}$ (95\%) in vertical [1].

As we all know that time synchronization of satellite clock plays a significant role in satellite navigations, accurate and reliable satellite clock offset parameters are the base of PNT service. Time synchronization error of satellite clock is mainly caused by the time transfer from the master station and its offset prediction. The clock prediction error depends on its frequency instability. Then the measurement, prediction and evaluation, of satellite clocks are very important for a satellite navigation system.

GPS operates a worldwide monitoring stations network, and includes six USAF stations, eleven NGA stations, and two IGS stations. Geodetic receivers are equipped in these stations to monitor the performance of satellite clocks. On 28 May, 2010, the first Block IIF satellite, designated SVN62/PRN25, was launched containing three atomic frequency standards, one DCBFS serial number 1010 (Cs 1010) and two RFS serial numbers 27 and $14(\mathrm{Rb} 27$ and $\mathrm{Rb} 14)$. The frequency stabilities of SVN62 Cs 1010 and Rb 27 are, respectively, $5 \times$ $10^{-14}$ and $7 \times 10^{-15}$ at 1 day in orbit. And the frequency stability of GPS Block IIR Rb is about $9 \times 10^{-15}$ at 1 day for October 2010 (all using NGA data) [2-4] while the frequency stability at 1 day for Galileo satellite clock is about $5 \times 10^{-14}$ for GIOVE A Rb clocks and $8 \times 10^{-15}$ for GIOVE B PHM $[5,6]$.

How about the performance of Beidou clocks in orbits? It is a very concerned question for many GNSS users. The system signals and observations of Beidou regional system are analyzed by Deutsches Zentrum fur Luft-und Raumfahrt (DLR) using a local monitoring network in March 2012. The short-term stability and middle-term stability of Beidou satellite clocks are analyzed and compared to other systems. Frequency stability of Beidou RAFS is about $7 \times 10^{-12} \sim$ $1 \times 10^{-11}$ at 1 second, Frequency stability of the best Beidou satellite clock is about $1 \times 10^{-13}$ at 1000 seconds, and GPS Block IIF is not worse than $1 \times 10^{-13}$ [7]. In the following sections, the time model of satellite clocks used by Beidou system is described; then the error source and uncertainty of the twoway radio time transfer (TWTT) are analyzed, which is used to measure clock differences between satellites and the uplink stations. Finally, the results and conclusions are detailed. 
TABLE 1: Basic information of Beidou satellite clocks.

\begin{tabular}{lcc}
\hline Num & Type (Num) & Date \\
\hline 03 & GEO-1 & 2010.1 .17 \\
04 & GEO-3 & 2010.6 .2 \\
05 & IGSO-1 & 2010.8 .1 \\
06 & GEO-4 & 2010.11 .1 \\
07 & IGSO-2 & 2010.12 .18 \\
08 & IGSO-3 & 2011.4 .10 \\
09 & IGSO-4 & 2011.7 .27 \\
10 & IGSO-5 & 2011.12 .2 \\
11 & GEO-5 & 2012.2 .25 \\
12 & MEO-3 & 2012.4 .30 \\
13 & MEO-4 & 2012.4 .30 \\
14 & MEO-5 & 2012.9 .19 \\
15 & MEO-6 & 2012.9 .19 \\
16 & GEO-6 & 2012.10 .25 \\
\hline
\end{tabular}

\section{Time Model of Satellite Clocks}

Considering the large-scale spacetime involved (about $1 \times$ $10^{5} \mathrm{~km}$ in space and several days, even several months or years in time) and the precision requirements $(1 \mathrm{~m}$, even $1 \mathrm{~cm}$, $1 \mathrm{~mm}$ level), the GNSS data process must be dealt with under the framework of relativity and quantum theory. Two kinds of conceptually different time scales are concerned in GNSS, proper times, and coordinate times. Essentially, for any two events, the observed space interval and time interval between them are dependent on the observer. The time readings given directly by ideal clocks located in satellites, stations, or observers are proper times. They are related to the observer, or to the spacetime environments of the clocks. This means that different observer has different clock due to its relative velocity and position in the gravitation field. In order to have a common time reference for all observers, we must choose a special observer and construct a reference system. A reference system contains a 3-dimensional space reference frame and a time reference. The former determines the spatial position (3 space coordinates) of an event and the latter gives the happening time, which is called coordinate time. For Earth satellites, a nonrotating geocentric reference system is used to describe their orbits. The reference time is usually TCG (the geocentric coordinate time) or TT (the terrestrial time) $[8,9]$.

The relationship between the proper time $\tau$ of satellite and the coordinate time TT (here noted by $t$ ) can be modeled as [8]

$$
\begin{aligned}
t= & {\left[1-\frac{\left(W_{0}-(3 / 2)(\mu / a)\right)}{c^{2}}\right]\left(\tau-\tau_{0}\right) } \\
& +\frac{2}{c^{2}} \sqrt{\mu a} \cdot e\left(\sin E-\sin E_{0}\right)
\end{aligned}
$$

where $\mu=\mathrm{GM}_{E}$ is the geocentric gravitation constant, $W_{0}$ the gravity potential of the geoid, $a$ the orbit main axis, $e$ the eccentricity, $E$ the real eccentric anomaly, and $c$ the speed of light, respectively. Then,

$$
\begin{aligned}
\tau-t= & {\left[\frac{W_{0}-\left((3 / 2)\left(\mathrm{GM}_{E} / a\right)\right)}{c^{2}}\right]\left(t-t_{0}\right) } \\
& -\frac{2}{c^{2}} \sqrt{a \mathrm{GM}_{E}} \cdot e \sin E
\end{aligned}
$$

If the satellite clock $T(t)$ is modeled as

$$
T(t)=\tau(t)+a_{0}+a_{1}\left(t-t_{0}\right)+a_{2}\left(t-t_{0}\right)^{2}+\xi(t) .
$$

in which $a_{0}, a_{1}$, and $a_{2}$ are clock offset parameters and $\xi(t)$ is the clock phase noise, the offset of satellite clock reffered to BDT can be written as

$$
\begin{aligned}
x(t) & \equiv T(t)-\operatorname{BDT}(t) \\
& =a_{0}+a_{1}\left(t-t_{0}\right)+a_{2}\left(t-t_{0}\right)^{2}+\Delta t_{\mathrm{grav}}^{p}+\xi(t)
\end{aligned}
$$

Here $\Delta t_{\text {grav }}^{p}$ is the periodic term of relativistic effect as follows:

$$
\Delta t_{\mathrm{grav}}^{p}=-\frac{2}{c^{2}} \sqrt{\mu a} e \sin E=-\frac{2 \vec{x}_{S} \cdot \dot{\vec{x}}_{S}}{c^{2}} .
$$

The relativistic effect must be taken into account for the evaluation of clock performance. If not, the stability of frequency will be influenced. The quasi-half-day periodical terms in Allan deviations of GPS clocks and Galileo clocks [2, 5], we guess, may be caused by this term.

\section{Two-Way Satellite Time Transfer and Error Analysis}

In Beidou system, TWTT between satellites and uplink stations is used for the satellitetime synchronization. The basic principle of TWTT is as follows. The satellites and stations generate and transmit pseudo-range signals controlled by their local clocks; then the uplink pseudo-range $\rho_{u}$ and downlink pseudo-range $\rho_{d}$ are measured by the satellites and the stations, respectively. The uplink pseudo-range and downlink pseudo-range can be written as

$$
\begin{aligned}
\rho_{u}\left(T_{S}^{r}\right)= & \left|\vec{x}_{S}\left(t_{S}^{r}\right)-\vec{x}_{R}\left(t_{R}^{e}\right)\right| \cdot \frac{1}{c}-\Delta T_{R}\left(t_{R}^{e}\right)+\Delta T_{S}\left(t_{S}^{r}\right) \\
& +\tau_{R}^{e}+\tau_{S}^{r}+\tau_{\text {tro }}+\tau_{\text {ion }}\left(f_{u}\right)+\tau_{\text {grav }}, \\
\rho_{d}\left(T_{R}^{r}\right)= & \left|\vec{x}_{R}\left(t_{R}^{r}\right)-\vec{x}_{S}\left(t_{S}^{e}\right)\right| \cdot \frac{1}{c}+\Delta T_{R}\left(t_{R}^{r}\right)-\Delta T_{S}\left(t_{S}^{e}\right) \\
& +\tau_{R}^{r}+\tau_{S}^{e}+\tau_{\text {tro }}+\tau_{\text {ion }}\left(f_{d}\right)+\tau_{\text {grav }},
\end{aligned}
$$

where $t_{S}^{r}$ and $t_{S}^{e}$ are time of reception and emission of the satellite signal; $t_{R}^{r}$ and $t_{R}^{e}$ are time of reception and emission of the station signal; $\Delta T_{S}$ and $\Delta T_{R}$ are satellite and station's clock offset; $\tau_{R}^{r}$ and $\tau_{R}^{e}$ are time delay of reception and emission of the station equipment; $\tau_{S}^{e}$ and $\tau_{S}^{r}$ are time delay of reception and emission of the satellite equipment; $\tau_{\text {tro }}$ and $\tau_{\text {ion }}$ are 
troposphere delay and ionosphere delay; $f_{u}$ and $f_{d}$ are uplink frequency and downlink frequency; $\tau_{\text {grav }}$ relativistic time delay caused by Earth gravitation.

The clock differences between satellites and stations are computed in the master station by using the uplink pseudoranges and the downlink pseudo-ranges. The satellite clock offset can be given by the observed uplink pseudo-range and downlink pseudo-range as follows:

$$
\begin{aligned}
\Delta T_{S}\left(t^{i}\right)= & \Delta T_{R}\left(t^{i}\right)+\frac{1}{2} \\
\times & \left\{\left[\rho_{u}\left(T_{S}^{i}\right)-\rho_{d}\left(T_{R}^{i}\right)\right]\right. \\
& \quad-\frac{1}{c}\left(\dot{\vec{x}}_{S}-\dot{\vec{x}}_{R}\right) \cdot \vec{n}_{R S}\left(\Delta T_{S}-\Delta T_{R}-\tau_{R S}\right) \\
& \left.+\Delta \tau_{R}-\Delta \tau_{S}-\Delta \tau_{\text {ion }}\right\}+\cdots,
\end{aligned}
$$

where

$$
\begin{gathered}
\Delta \tau_{R} \equiv \tau_{R}^{r}-\tau_{R}^{e}, \\
\Delta \tau_{S} \equiv \tau_{S}^{r}-\tau_{S}^{e}, \\
\Delta \tau_{\text {ion }} \equiv \tau_{\text {ion }}\left(f_{u}\right)-\tau_{\text {ion }}\left(f_{d}\right), \\
\vec{n}_{R S} \equiv \frac{\left(\vec{x}_{S}-\vec{x}_{R}\right)}{\left|\vec{x}_{S}-\vec{x}_{R}\right|} .
\end{gathered}
$$

The random error of satellite clock difference includes the noise of pseudo-range observable and the satellite clock phase noise. In short term $(\leq 1000 s)$, the influence of the frequency drift and phase noise of satellite clock to clock offset can be neglected. So the uncertainty of type A of satellite-board clock offset measurement can be calculated by the fluctuation of clock difference. Analysis shows that the uncertainty of type $\mathrm{A}$ is less than $0.3 \mathrm{~ns}[10]$. In middle or long term $(\geq 10000 \mathrm{~s})$, the influence of the pseudo-range noise can be neglected, and the results of the Allan variance of satellite clocks are reliable.

\section{Performance Evaluation of Beidou Satellite Clocks in Orbit}

Satellites that include GEO satellites of serial number 03,04 , 06 , and 11, IGSO satellites of serial number 07, 08, 09 and 10, and MEO satellites of serial number 13 and 14 are evaluated. In order to ensure the reliability of the evaluation result, the time interval of satellite clock data is no less than 15 days. The time scale reference for analysis is the high performance hydrogen clock in ground.

Figures 1,2, and 3 show the linear residuals and secondorder polynomial residuals of the observed satellite clock offsets.

The green curves are plots of the linear residuals of satellite clocks. All of the linear residual of GEO-3, GEO-4, and IGSO-2 are smooth, which mean that the rubidium clocks have significant frequency drifts. The blue curves are the second-order polynomial residuals of satellite clocks, which

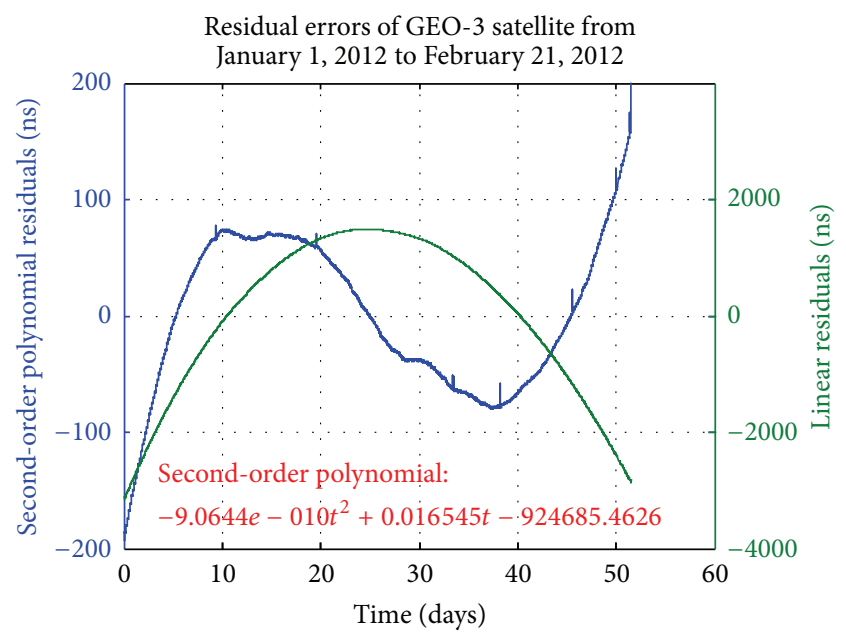

Figure 1: Residual of GEO-3 satellite clock.

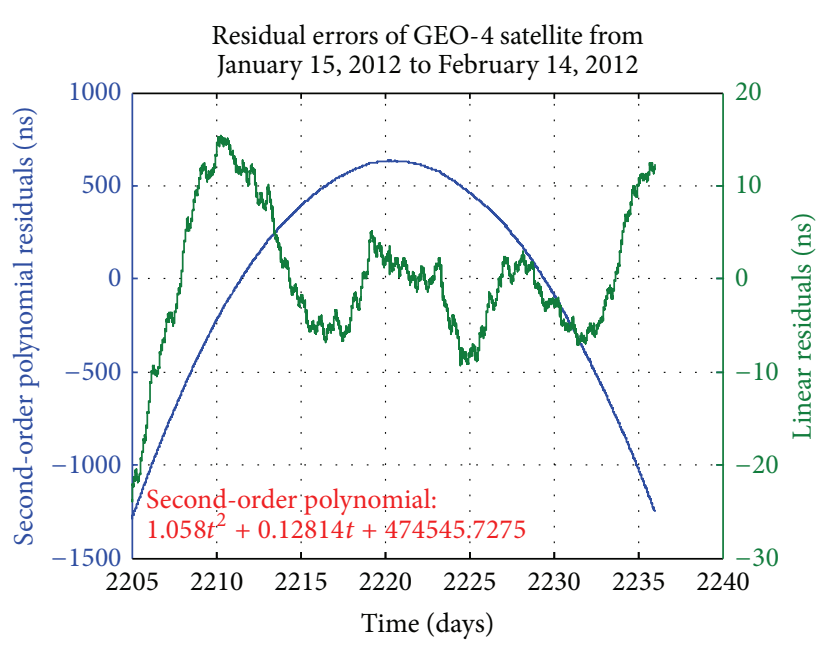

Figure 2: Residual of GEO-4 satellite clock.

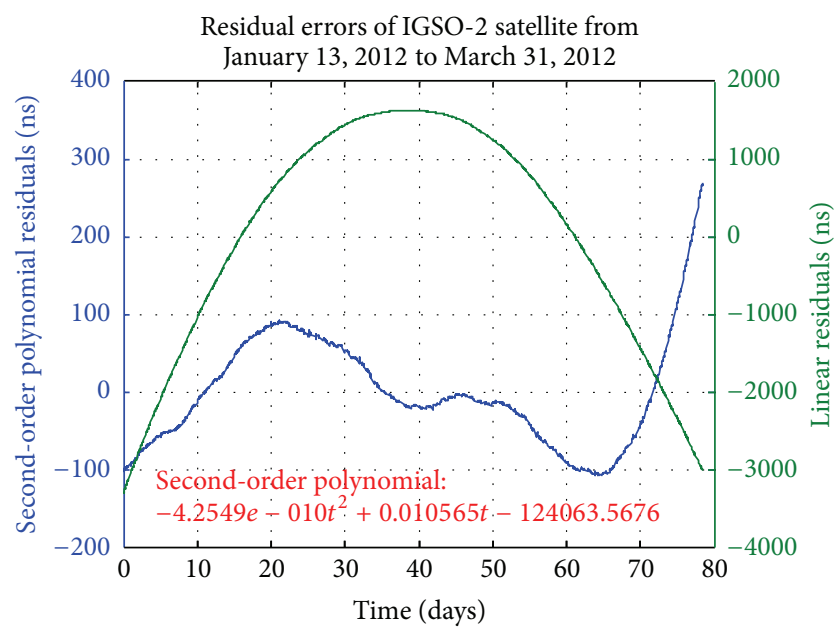

FIgURE 3: Residual of IGSO-2 satellite clock. 
TABLE 2: Frequency stability of Beidou system satellite clocks.

\begin{tabular}{|c|c|c|c|c|c|c|c|c|c|}
\hline & GEO-1 & GEO-3 & GEO-4 & GEO-5 & IGSO-1 & IGSO-2 & IGSO-3 & IGSO-4 & IGSO-5 \\
\hline $\begin{array}{l}\text { Stability } \\
\text { (10000 s) }\end{array}$ & $7.31 \times 10^{-14}$ & $5.52 \times 10^{-14}$ & $7.58 \times 10^{-14}$ & $9.17 \times 10^{-14}$ & $8.13 \times 10^{-14}$ & $5.95 \times 10^{-14}$ & $7.94 \times 10^{-14}$ & $8.53 \times 10^{-14}$ & $8.98 \times 10^{-14}$ \\
\hline $\begin{array}{l}\text { Stability } \\
\text { (1 day) }\end{array}$ & $6.71 \times 10^{-14}$ & $2.90 \times 10^{-14}$ & $3.83 \times 10^{-14}$ & $5.66 \times 10^{-14}$ & $9.38 \times 10^{-14}$ & $3.07 \times 10^{-14}$ & $2.53 \times 10^{-14}$ & $3.91 \times 10^{-14}$ & $4.45 \times 10^{-14}$ \\
\hline
\end{tabular}

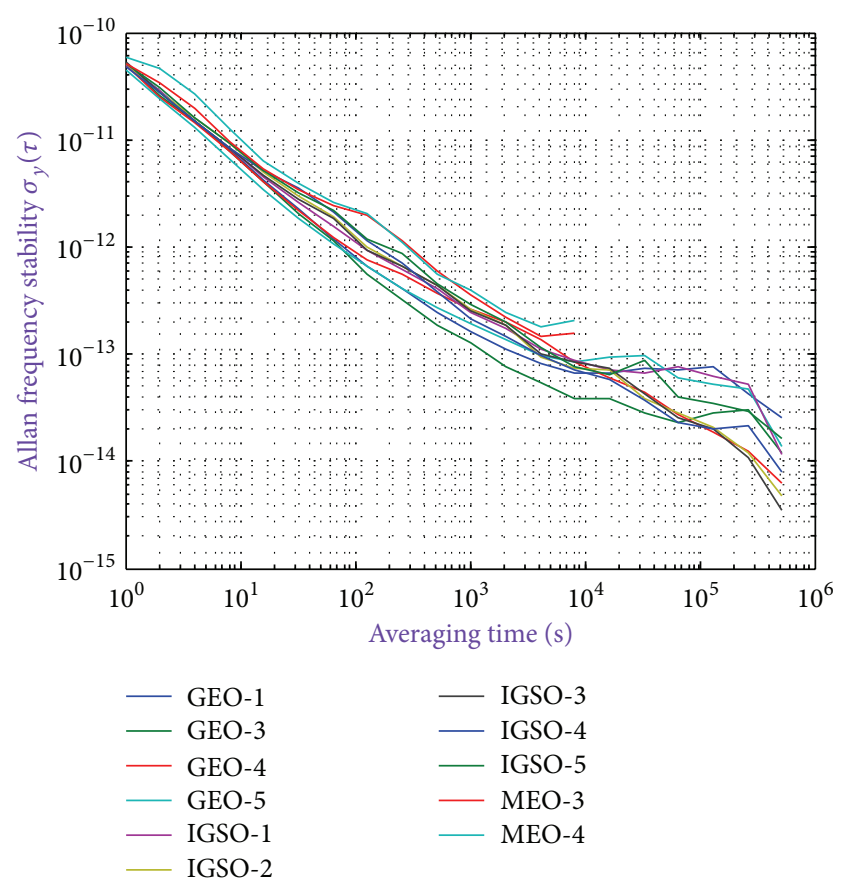

Figure 4: Frequency stability of Beidou system satellite clocks.

demonstrate that the frequency drafts are changing slowly and the rubidium clocks in orbit have high-level noise characteristic, such as flick and random walk.

The frequency stability of Beidou satellite clocks is evaluated by use of the overlapping Allan deviation. Figure 4 shows plots of the frequency stability of Beidou system satellite clocks. Table 2 shows the frequency stability at a sample time of 10000 seconds and 1 day.

The frequency stability of Beidou satellite clocks is of the level of $10^{-14}$ at a sample time of 10000 seconds and 1 day. The frequency stability at a sample time of 10000 seconds is about $5.95 \sim 9.17 \times 10^{-14}$, and that at a sample time of 1 day is about $2.53 \sim 9.38 \times 10^{-14}$.

Figure 5 gives the comparison of the clock performances in orbit and in the ground vacuum. The results show that the performances in orbits are conformable with those in ground. As a whole, the results in orbit are a little better than those in ground.

\section{Conclusion}

The long-term evaluation for Beidou satellite clocks has been done using TWTT between satellites and stations. The results

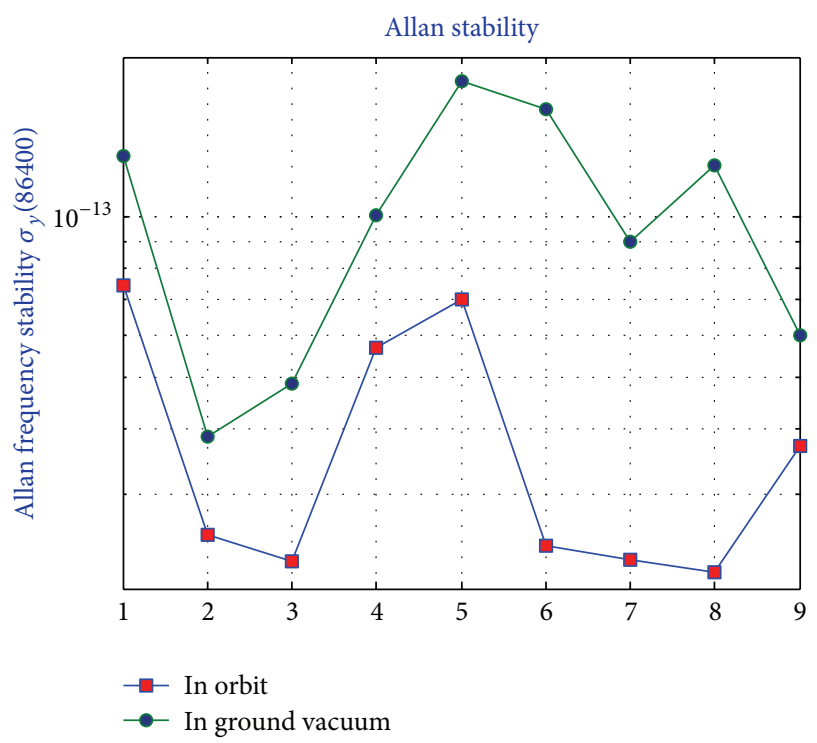

FIGURE 5: Clock day stabilities in orbit and in ground vacuum pots.

show that the performance of satellite clock is steady and in good condition. The frequency stabilities at a sample time of $10000 \mathrm{~s}$ and 1 day for all the satellite clocks are better than $1.0 \times$ $10^{-13}$. It means that the performance of Beidou satellite clocks in orbit is consistent with the ground test, and the results in orbit are a little better than those in ground vacuum.

\section{Acknowledgments}

The authors wish to thank the Editor Sandro M. Radicella, the Editorial Assistant Ms. Joanna, and the anonymous reviewers whose comments helped improve this paper enormously.

\section{References}

[1] H. Qiaohua, "Development of Beidou navigation satellite system," in Proceedings of the 5th Meeting of International Committee on GNSS (ICG-5 '12), Beijing, China, 2012.

[2] F. Vannicola, R. Beard, J. White, and K. Senior, "GPS Block IIF atomic frequency standard analysis," in Proceedings of the 42th Annual Precise Time and Time Interval (PTTI) Meeting, pp. 181196, 2010.

[3] J. Oaks, J. A. Buisson, and M. M. Largay, "A summary of the GPS constellation clock performance," in Proceedings of the 39th Annual Precise Time and Time Interval (PTTI) Meeting, pp. 119130, 2007.

[4] D. M. Manning and C. P. Petersen, "AF/NGA GPS monitor station high-performance cesium frequency standard stability 
2007/2008: from NGA kalman filter clock estimates," in Proceedings of the 40th Annual Precise Time and Time Interval (PTTI) Meeting, pp. 335-348, 2008.

[5] P. Waller, F. Gonzalez, S. Binda et al., "The in-orbit performances of GIOVE clocks," IEEE Transactions on Ultrasonics, Ferroelectrics, and Frequency Control, vol. 57, no. 3, pp. 738-745, 2010.

[6] P. Waller, F. Gonzalez, and S. Binda, "Long-term performance analysis of giove clocks," in Proceedings of the 42th Annual Precise Time and Time Interval (PTTI) Meeting, pp. 171-180, 2010.

[7] O. Montenbruck, A. Hauschild, P. Steigenberger, U. Hugentobler, P. Teunissen, and S. Nakamura, "Initial assessment of the compass/beidou-2 regional navigation satellite system," GPS Solutions, vol. 17, no. 2, pp. 211-222, 2013.

[8] H. Chunhao, "Time measurement within the frame of relativity," Progress in Astronomy, vol. 20, no. 2, pp. 107-113, 2002.

[9] H. Chunhao, C. Zhiwu, L. Yuting, L. Li et al., "Time synchronization and performance evaluation of beidou satellite clocks," in Proceedings of the 3rd China Satellite Navigation Conference, 2012.

[10] L. Liu, L.-F. Zhu, C.-H. Han, X.-P. Liu, and C. Li, "The model of radio two-way time comparison between satellite and station and experimental analysis," Chinese Astronomy and Astrophysics, vol. 33, no. 4, pp. 431-439, 2009. 

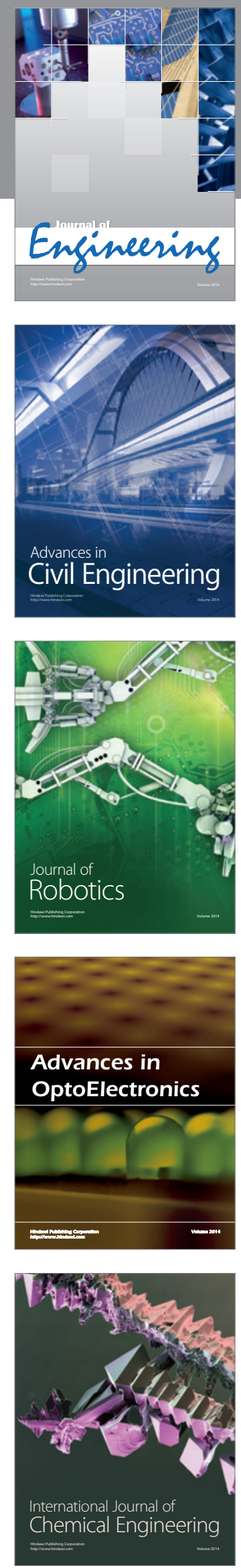

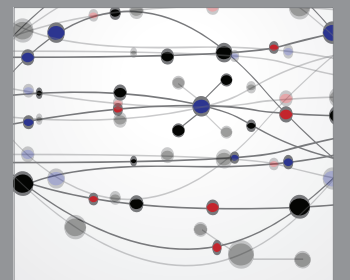

The Scientific World Journal
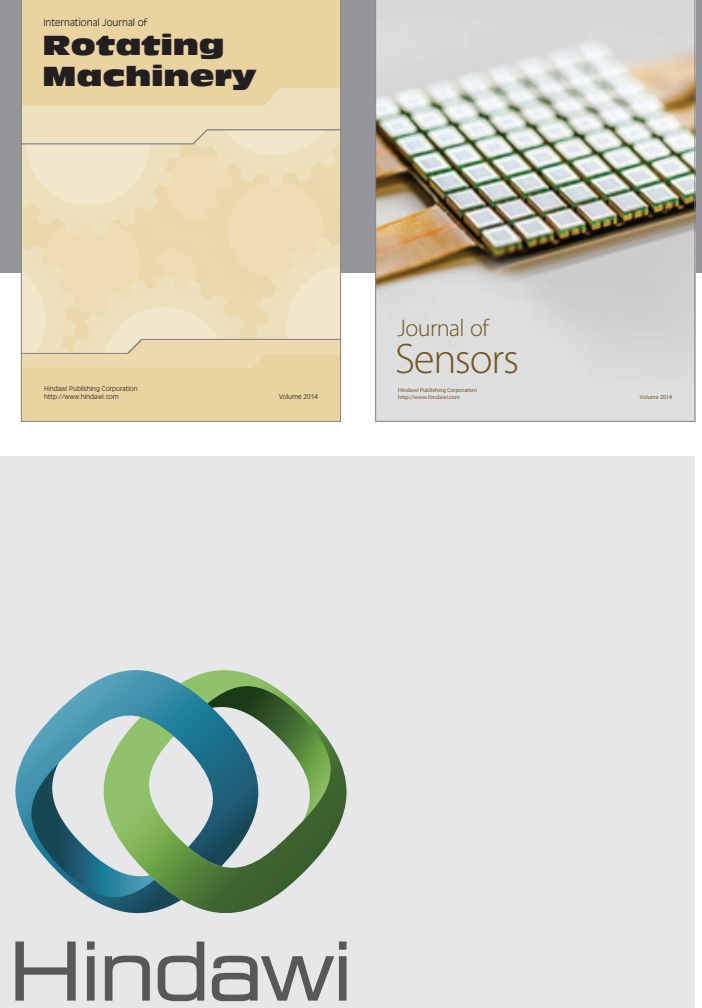

Submit your manuscripts at http://www.hindawi.com
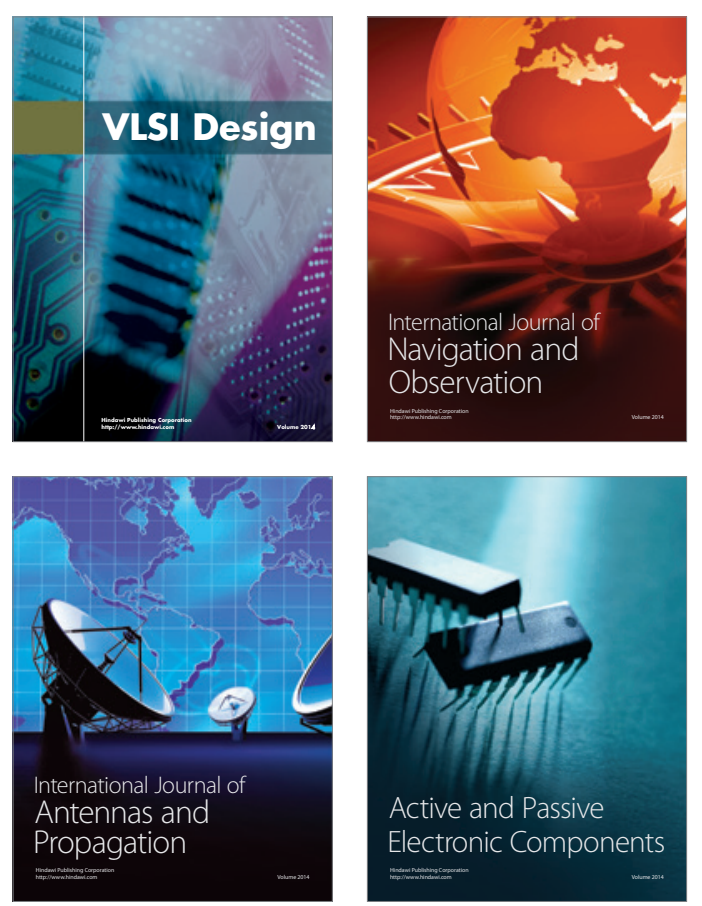
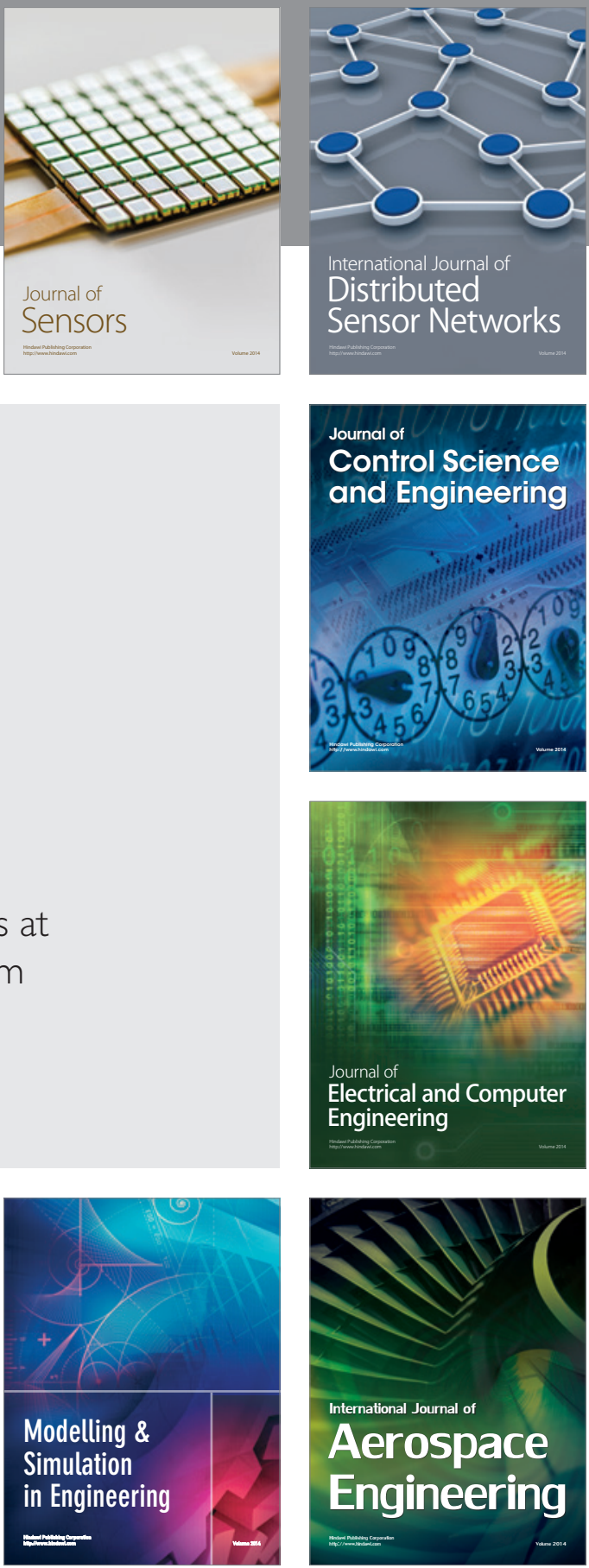

Journal of

Control Science

and Engineering
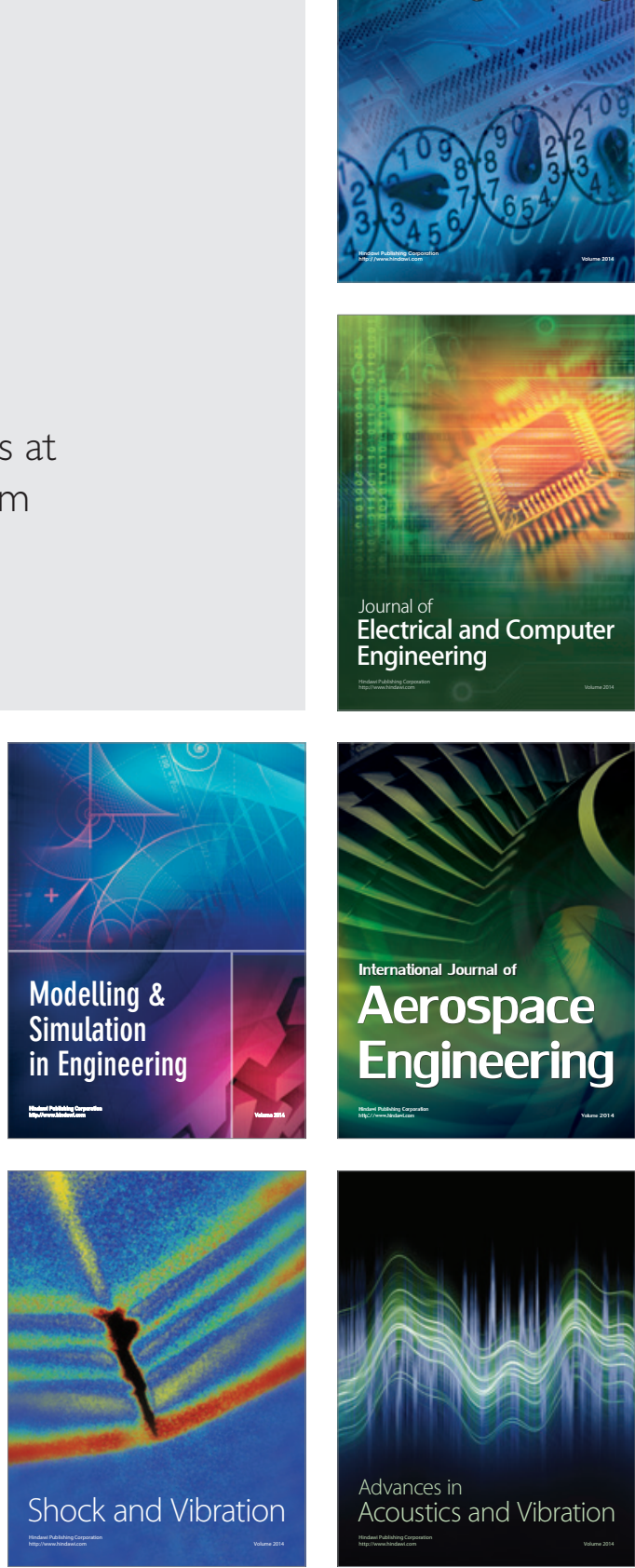